\title{
MEASUREMENT OF THE COSMIC X-RAY BACKGROUND \\ IN THE 25-200 keV RANGE
}

\author{
D. BRINI, F. FULIGNI, and E. HORSTMAN-MORETTI \\ Space Research Group of the Lab.TESRE of the C.N.R., \\ Institute of Physics of Bologna University, Italy
}

An apparatus to study the cosmic X-ray background was launched aboard an ESRO Skylark rocket on May 22, 1967 from the Perdas de Fogu range in Sardinia.

The basic detector consisted of a $\mathrm{NaI}(\mathrm{Tl})$ phoswich, $5 \mathrm{~cm}$ in diameter and $1.27 \mathrm{~cm}$ thick, surrounded by both passive (lead) and active (plastic scintillator) cylindrical collimators.

Four of these were placed at $45^{\circ}$ from the rocket axis and arranged symmetrically around it, one of them being completely screened to give a direct evaluation of the instrumental background, while the large aperture (about $30^{\circ} \mathrm{FWHM}$ ) of the other three allowed a wide scan of the sky.

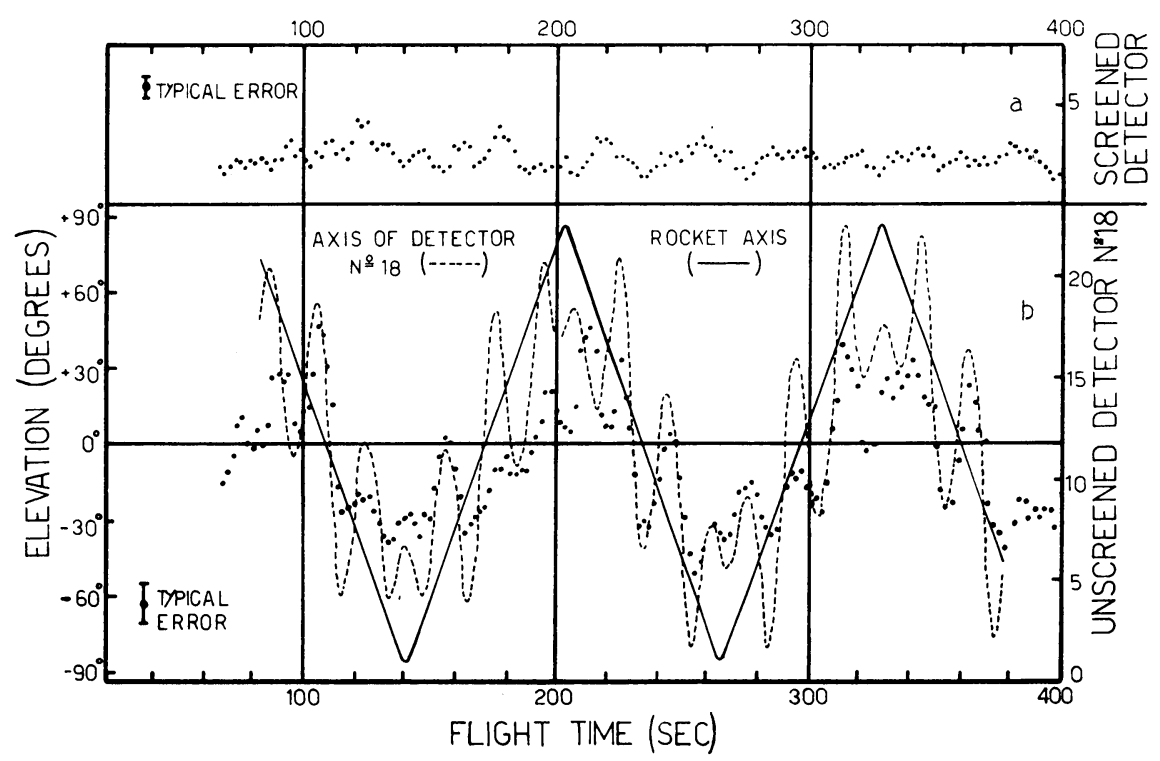

Fig. 1. (a) Integral (25-100 keV) counting rate of the screened detector as a function of time after launch. - (b) Integral (25-200 keV) counting rate of one of the unscreened detectors (No. 18) as a function of time after launch. Counting rates are obtained by averaging over periods of 8 sec. In contrast of what happens to the screened detector, which keeps constant, we note the correlation existing between the counting rate of the detector (No. 18), and the elevation of the detector axis (dotted line) and the rocket axis (full line).

The events from each detector were analyzed in four energy channels (nominally $25-40,40-70,70-110$, and $110-210 \mathrm{keV}$ ) for which the product of the efficiency and the geometric factor gave, respectively, $6.4,7.2,7.3$ and $6.5 \mathrm{~cm}^{2} \mathrm{sr}$. 
The flight, started at $0107 \mathrm{UT}$ and reaching $192 \mathrm{~km}$, was a successful one but for malfunctions of the longitudinal magnetometer and lunar cells, which caused doubts and delay in the attitude determination. We believe, however, that our data behaviour itself, as shown in Figure 1 for one of the detectors together with the corresponding elevation vs. time, provides a satisfactory check of the final aspect solution.

While the screened detector counting rate stays constant after cone ejection, the three unscreened detectors show a consistent variation with elevation in the lower

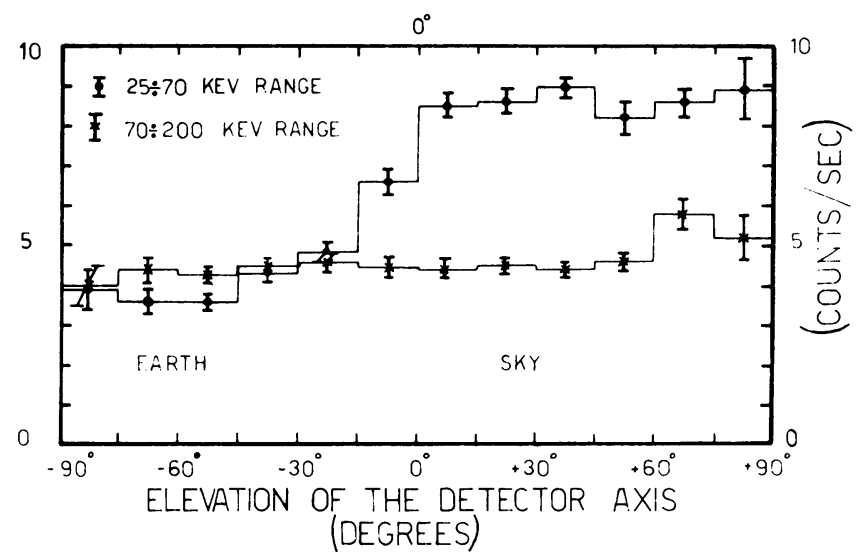

Fig. 2. Counting rates averaged over the three unscreened detectors as a function of the elevation of the detector axis. Please note the sky-earth asymmetry for the counting rate in the $20-70 \mathrm{keV}$ energy range. This shows that the spectrum of the cosmic X-Ray background is steeper than that of the albedo X-rays.

energy channels. This can also be seen from Figure 2 where the data from all three detectors have been considered.

Because the X-ray horizon is about $105^{\circ}$ from the zenith at our altitudes and energies, the data with positive elevation have been considered to be of extra-atmospheric origin and were analyzed with respect to celestial coordinates.

Because of the motion of the despun rocket, most of the sky above the horizon was seen, although the coverage was not uniform. Many X-ray sources were scanned, namely those in the Scorpio-Sagittarius region and the Cygnus region.

Our counting rate for both zones showed increases well above the general average. We also saw an increase from the direction of M87, but it was not statistically significant. Further analysis is needed for the sources, although the wide aperture makes it a difficult and ambiguous task. We could not, however, include the counting rates from these regions in our collection of background data, even if this reduces the explored area and statistics (especially at low galactic latitudes).

So, we did not consider data for which the detector's axis direction was less than $18^{\circ}$ away from one of the ten major sources, taking as a parameter the low energy fluxes quoted by NRL (1966). We also excluded the M87 region even though it would have had a small effect on the background average. 


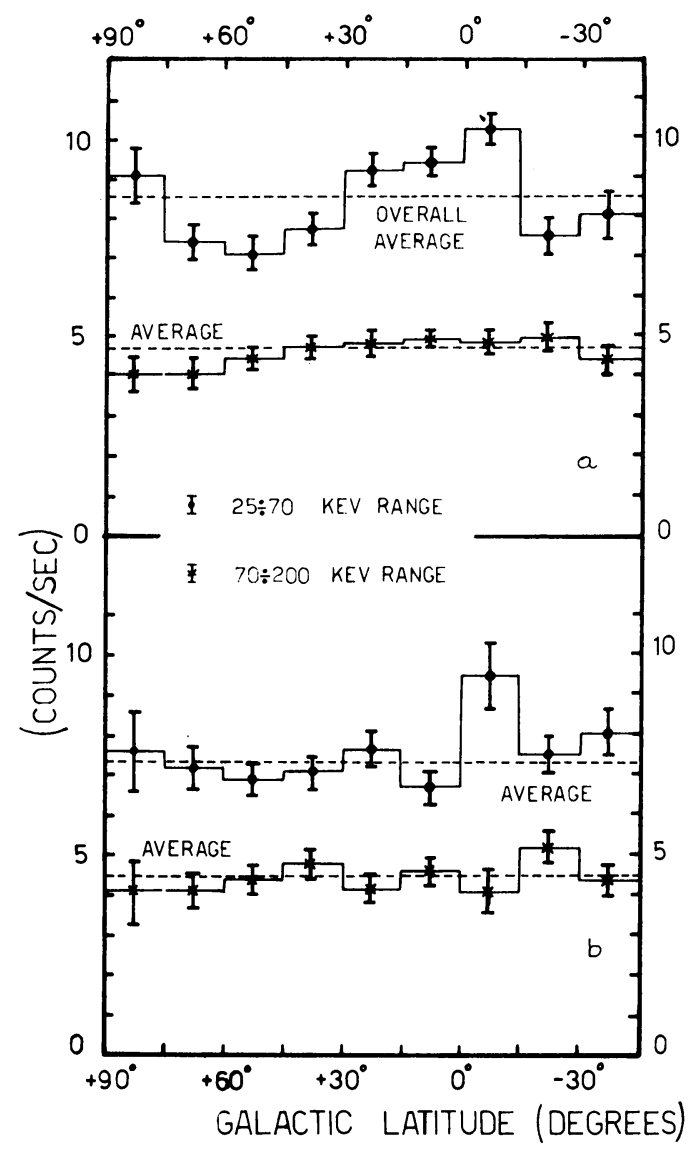

Fig. 3. Counting rates of the three unscreened detectors as a function of the galactic latitude. (a) All data referring to elevation $>0^{\circ}$. - (b) The same but excluding the regions of X-ray sources.

Figure 3 shows the results of the analysis vs. galactic latitude, both including and excluding data from the 'hot' regions. For the latter, no systematic trend appears in the background behaviour. We compared the total counting rates (corrected for instrumental background) of the galactic equator (from $-15^{\circ}$ to $+15^{\circ}$ ) and the pole (above $+60^{\circ}$ ) to evaluate the anisotropy as given by $A=(E-P) /(E+P)$.

We obtained $A=0.03 \pm 0.04$ thus setting an upper limit of $11 \%$ at the $2 \sigma$ level for the anisotropy in our energy range.

The counting rates outside the source regions, corrected for efficiency, channel width, instrumental background and geometric factor, give intensities of $(1.29 \pm 0.04) \mathrm{cm}^{-2} \mathrm{sr}^{-1}$ and $(0.76 \pm 0.04) \mathrm{cm}^{-2} \mathrm{sr}^{-1}$ respectively for sky and earth fluxes, while the corresponding energy spectra are plotted in Figure 4.

Only statistical errors are shown, to which an uncertainty of about $10 \%$ over the energy thresholds should be added. 


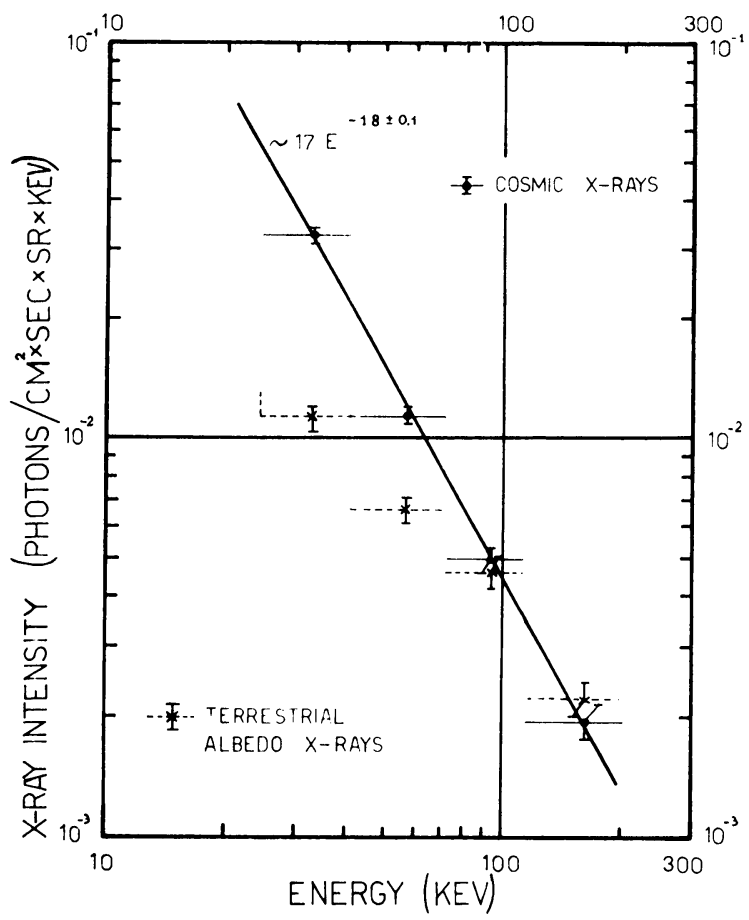

Fig. 4. Energy spectrum of the diffuse cosmic X-ray background as measured in this experiment. Also shown is the spectrum of the terrestrial X-rays albedo (dotted line).

As can be seen, the good agreement of the data with a power law $E^{-1.8 \pm 0.1}$ does not call for any break or substantial bend in the spectrum.

Since the subtraction of the instrumental background was negligible for all but the higher energy channel, we give more importance, however, to the results from the first three channels. If there is indeed an abrupt change in the slope, we believe it must be outside the range $25-110 \mathrm{keV}$.

The albedo spectrum appears different from what has been measured inside the atmosphere.

We think that it shows a contribution of scattered primary X-rays, which should be taken into account in the extrapolations necessary in balloon measurements.

This could be a reason, together without direct measurement of the instrumental background, for the disagreement between our results and other measurements made in the same energy region.

Looking at the data for all energies studied, one feels, however, that an overall agreement could be found with a power law spectrum similar to our result. 